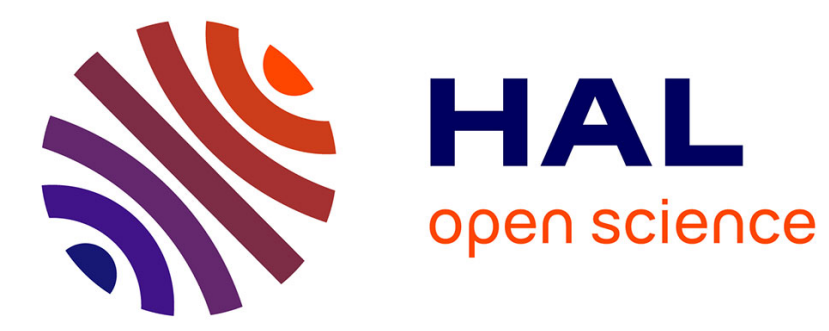

\title{
Partenariats en santé mentale à partir de l'école
}

Sandrine Bonneton, Isabelle Maillard, Thibaud Pombet, Livia Velpry

\section{To cite this version:}

Sandrine Bonneton, Isabelle Maillard, Thibaud Pombet, Livia Velpry. Partenariats en santé mentale à partir de l'école. Rhizome , 2020, Nº7 (4), pp.12-13. 10.3917/rhiz.078.0012 . halshs-03231081

\section{HAL Id: halshs-03231081 https://shs.hal.science/halshs-03231081}

Submitted on 27 Jan 2022

HAL is a multi-disciplinary open access archive for the deposit and dissemination of scientific research documents, whether they are published or not. The documents may come from teaching and research institutions in France or abroad, or from public or private research centers.
L'archive ouverte pluridisciplinaire HAL, est destinée au dépôt et à la diffusion de documents scientifiques de niveau recherche, publiés ou non, émanant des établissements d'enseignement et de recherche français ou étrangers, des laboratoires publics ou privés. 


\section{Partenariats en santé mentale à partir de l'école}

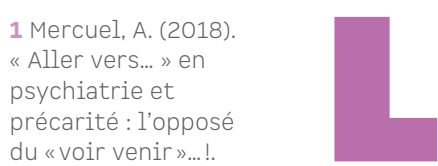

a précarisation des conditions de vie et le développement des processus de vulnérabilisation des populations ont conduit à faire de " l'aller vers ${ }^{1}$ » un enjeu majeur Rhizome, 68(2), 3-4.

2 Maillard, I. (2011) Lignes de force de l'évolution de politiques publiques relatives à la santé mentale des adolescents. Santé publique, 23(HS), 127-140.

3 Lahire, B. (dir.) (2019). Enfances de classe. De l'inégalité parmi les enfants. Paris : Seuil.

4 Moro, M-R et

Brison, J.-L. (2016)

Mission Bien-être

et santé des jeunes

et santé des jeunes.

l'Éducation nationale

ministère de la Santé,

ministère de la ville,

ministère des Affaires

sociales et de la Santé

5 cette recherche interventionnelle portée par le Groupe de coopération sanitaire pour la recherche et la formation en santé mentale et coordonnée par Sandrine Bonneton (pédo-psychiatre) associe plusieurs sociologues : Isabelle Maillard

(Eceve), Aude Béliard.

Thibaud Pombet et Livia Velpry (Cermes) et Jean-Sébastien Eideliman (Cerlis)

des politiques publiques afin de favoriser l'accès à la prévention aux soins en santé mentale ${ }^{2}$. Cette approche prend une acuité particulière dans le cas des enfants dont les difficultés s'expriment en premier lieu à l'école ${ }^{3}$. Le développement de « nouvelles alliances ${ }^{4}$ » entre structures sanitaires et écoles est encouragé pour dépasser les logiques de cloisonnement institutionnel, qui limitent l'accompagnement des enfants dont les difficultés sont souvent multidimentionnelles et situées à l'intersection de différents champs d'action. À partir d'une recherche interventionnelle qui vise à construire des partenariats entre écoles, centres médico-psychologiques (CMP) et conseil locaux en santé mentale (CLSM) sur plusieurs sites en France, cet article examine les enjeux de l'« aller vers » réactualisés par la

\section{La recherche Parpsych-Ed 5 et les enjeux} des partenariats locaux écoles-CMP

La mise en œuvre de partenariats et la proposition d'interventions précoces à partir du milieu de vie des enfants comme l'école visent à éviter leur évolution vers des formes graves et à réduire des inégalités sociales, scolaires et de santé, souvent intriquées. Ces alliances et dispositifs soulèvent toutefois de nombreuses questions et leur légitimité fait débat parmi les professionnel-le.s. La recherche Parpsych-Ed part d'une crise sanitaire de la COVID-19. interrogation sur des transformations à envisager au niveau du secteur de psychiatrie infanto-juvénile afin d'apporter des réponses aux enfants dont les problématiques « frontières » interrogent et mettent en difficulté les acteur.rice-s scolaires. Il s'agit de privilégier des interventions à partir de l'école à un moment où les problèmes ne sont pas encore véritablement constitués ni étiquetés, et de favoriser, grâce à des interventions précoces, l'accès aux soins s'ils sont nécessaires.

Réunissant sociologues, chercheurs en santé publique et pédopsychiatres, l'équipe propose d'accompagner, analyser et évaluer la construction de partenariats locaux entre le secteur infanto-juvénile et un établissement scolaire autour de la mise en œuvre d'interventions pluriprofessionnelles, transversales aux deux institutions, en direction d'enfants de 3 à 8 ans - scolarisés de la petite section de maternelle jusqu'au CE1 - présentant des difficultés dont la composante psychique reste le plus souvent hypothétique. Parallèlement à ce volet interventionnel, une étude sociologique est menée pour analyser et comparer les contextes et conditions de leur déploiement dans les différents territoires concernés.

Les équipes des CMP qui participent à cette recherche l'ont fait sur la base du volontariat. Le choix des écoles pour déployer le partenariat, fait en concertation avec les inspecteur.rice.s de l'Éducation nationale et les directeur.rice.s d'école, s'est porté majoritairement sur des écoles situées en zones réseau d'éducation prioritaire (REP) ou réseaux d'éducation prioritaire renforcés (REP +), croisant les quartiers prioritaires de la ville. Dans ces écoles, les enseignant·e.s, plus qu'ailleurs, peuvent avoir le sentiment qu'ils.elles exercent dans des situations limites et estiment que bon nombre d'enfants ne vont pas bien (jusqu'à

$90 \%$ des élèves par classe pour certains sites). Sur ces territoires, les écoles restent parfois la seule institution que les familles peuvent solliciter pour des problèmes qui dépassent bien souvent le cadre scolaire. Enfin, en l'absence d'investigation clinique, la question du « besoin de soins " peut laisser perplexes certains parents qui ne comprennent pas l'insistance de leurs interlocuteur.rice.s scolaires pour qu'ils accompagnent leur enfant vers des soins, conduisant parfois à des tensions avec les équipes.

\section{Les effets du confinement sur les liens} entre familles, enfants et professionnel-les À partir d'entretiens téléphoniques individuels et d'échanges collectifs par visioconférences conduits avec les acteurs des écoles et des CMP des principaux sites de la recherche, la période de confinement liée à la pandémie de la COVID-19 a été l'occasion de saisir l'impact de cette crise sur la réorganisation des services et des pratiques professionnelles. Sans surprise, cette période a agi comme un amplificateur des inégalités sociales. Dans les territoires les plus marqués par la pauvreté, la préoccupation des acteur.rice-s de l'école et du soin n'était pas seulement d'assurer une continuité de lien, pédagogique ou thérapeutique. Elle était aussi de s'assurer que les familles pouvaient subvenir à des besoins élémentaires concernant leur alimentation, leurs conditions de vie, leurs déplacements essentiels ou encore la stimulation des enfants. Face aux situations les plus préoccupantes, diverses actions ont joué un rôle de relais (appels téléphoniques quotidiens, distribution de colis alimentaires, de jouets, impressions d'attestations de sortie).

Dans ce contexte, le renouvellement des pratiques professionnelles a contribué à produire des rapprochements inattendus avec certains parents ou enfants, 
contribuant, selon les professionnelle.s à faire « tomber des barrières " jusque-là peu ou pas perçues. L'usage d'outils numériques a favorisé une prise de parole de certaines familles qui s'expriment très peu dans les instances ordinaires de l'école. L'école à la maison a nécessité de "faire alliance " avec les parents, de les considérer comme des partenaires de l'activité scolaire, ce qui a été vécu comme très valorisant par ces derniers. Certains enfants, dont plusieurs en difficulté à l'école, ont connu une évolution favorable tant au niveau des apprentissages que dans la relation avec leur enseignant.e, surprenant les équipes par leurs capacités expressives et scolaires.

Si l'utilisation de ces outils numériques s'est heurtée aux inégalités d'accès - sociales et territoriales -, elle a aussi parfois permis de contourner des problèmes d'organisation du quotidien des professionnelle.s et des familles, par exemple dans des territoires mal desservis par les transports publics, ou encore lorsque lieux de travail, écoles et CMP sont géographiquement éloignés. Dans certaines écoles et CMP, la pérennisation des échanges entre professionnel-le-s et familles par visioconférence et téléphone est envisagée comme un moyen d'établir un lien privilégié, dans un souci de différenciation pédagogique ou d'alliance thérapeutique pour réduire les barrières à l'accès aux soins.

S'il est trop tôt pour apprécier les effets du confinement ou l'impact de ces pratiques sur l'évolution des situations d'enfants en difficulté, il est certain que le contexte pandémique a fait bouger certaines lignes au niveau des relations entre professionnel.le.s et familles. Le déploiement de partenariats à l'interface école-CMP sur des territoires fortement marqués par les inégalités sociales et économiques pourrait dès lors s'appuyer sur ces expériences inscrites dans une dynamique de l'« aller vers".

\section{L'《aller vers », comment et avec quels objectifs?}

Dans tous les sites participant à la recherche, les professionnel-le.s envisagent le déploiement d'interventions précoces à l'interface école-CMP comme intimement lié aux conditions socio-économiques de certains territoires où les familles, plus qu'ailleurs, sont soumises à des contraintes sociales qui pèsent sur le développement de leurs enfants ${ }^{6}$. Cette approche de l'« aller vers », développée dans le champ de la précarité auprès des grands exclus, semble ainsi pertinente pour penser les problèmes psychiques des enfants, tels qu'ils se posent aux professionnel-le.s et aux chercheur.e.s. Elle permet de répondre à des problèmes complexes, intriqués, difficiles à démêler et aussi d'envisager des stratégies thérapeutiques différenciées via la mise en œuvre de modes d'entrée et de délivrance des soins qui tiennent davantage compte des contextes dans lesquels évoluent les enfants et des attentes de leurs parents. Dans cette perspective, il s'agit d'envisager ce qui, à partir de l'école, peut favoriser ou, au contraire, limiter une demande de soins sur les structures pédopsychiatriques ad hoc, en considérant les représentations de la santé mentale qu'ont les professionnel.le.s de l'école et les familles, mais aussi plus globalement le rapport des parents aux institutions et leurs attentes vis-à-vis de l'école.

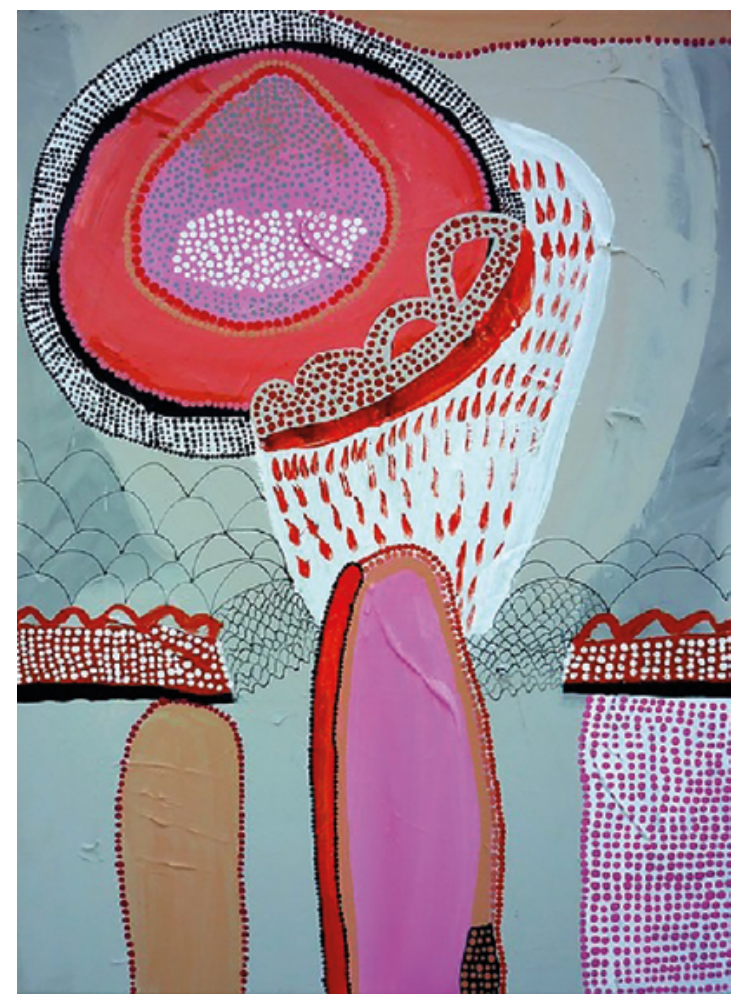

Aux difficultés rencontrées par les enfants et leurs familles viennent s'ajouter, dans les zones les plus défavorisées, de fortes inégalités au niveau des ressources éducatives, avec parfois un turn over important des professionnel-le· $\mathrm{s}^{7}$.

Dans ces situations, les partenariats ont aussi pour objectif de soutenir les équipes éducatives face aux problèmes qui traversent leur institution et d'offrir des espaces de réflexion qui redessinent les frontières d'actions à l'intérieur et à l'extérieur du système sanitaire. Le défi consiste à trouver l'équilibre entre approche individuelle et collective, permettant d'accompagner depuis l'école les enfants en difficulté sans pour autant verser dans une individualisation excessive, voire une médicalisation des réponses. La recherche sur laquelle s'appuie cet article favorise le développement de partenariats et, via la participation des CLSM, participe à redéfinir les politiques locales de santé mentale, en faisant une plus grande place à l'Éducation nationale, jusqu'à maintenant faiblement intégrée et impliquée au sein de ces instances ${ }^{8}$.

\section{conclusion}

Dans le contexte pandémique actuel, avec le risque d'aggravation des inégalités qu'il fait peser, le développement de pratiques à l'interface écoleCMP promouvant l'« aller vers » semble plus crucial encore. Éviter que tous les problèmes ne se cristallisent à l'école et faciliter un accompagnement adapté pour le plus grand nombre représente néanmoins un véritable défi pour les professionnel.le.s. Sile contexte de restriction budgétaire et de baisse des effectifs, parallèle à l'augmentation des files actives en pédopsychiatrie, est peu favorable à leur développement, ces approches semblent pourtant particulièrement pertinentes pour aider les enfants les plus vulnérables psychiquement compte tenu du poids que la crise actuelle fait peser sur leurs familles. $)$ 